\title{
An Adaptive Fuzzy C-Means Algorithm for Improving MRI Segmentation
}

\author{
Elnomery Allam Zanaty \\ Mathematics Department, Faculty of Science, Sohag University, Sohag, Egypt \\ Email: zanaty22@yahoo.com
}

Received September 20, 2013; revised October 20, 2013; accepted October 27, 2013

Copyright (C) 2013 Elnomery Allam Zanaty. This is an open access article distributed under the Creative Commons Attribution License, which permits unrestricted use, distribution, and reproduction in any medium, provided the original work is properly cited.

\begin{abstract}
In this paper, we propose new fuzzy c-means method for improving the magnetic resonance imaging (MRI) segmentation. The proposed method called "possiblistic fuzzy c-means (PFCM)" which hybrids the fuzzy c-means (FCM) and possiblistic c-means (PCM) functions. It is realized by modifying the objective function of the conventional PCM algorithm with Gaussian exponent weights to produce memberships and possibilities simultaneously, along with the usual point prototypes or cluster centers for each cluster. The membership values can be interpreted as degrees of possibility of the points belonging to the classes, i.e., the compatibilities of the points with the class prototypes. For that, the proposed algorithm is capable to avoid various problems of existing fuzzy clustering methods that solve the defect of noise sensitivity and overcomes the coincident clusters problem of PCM. The efficiency of the proposed algorithm is demonstrated by extensive segmentation experiments by applying them to the challenging applications: gray matter/white matter segmentation in magnetic resonance image (MRI) datasets and by comparison with other state of the art algorithms. The experimental results show that the proposed method produces accurate and stable results.
\end{abstract}

Keywords: Fuzzy Clustering; Possiblistic C-Means; Medical Image Segmentation

\section{Introduction}

Clustering is one of the most popular classification methods and has found many applications in pattern classification and image segmentation [1-6]. With increasing use of magnetic resonance imaging (MRI) for diagnosis, treatment planning and clinical studies, it has become almost necessary for radiological experts to make clinical diagnosis and treatment planning by using computers. Medical images tend to suffer much more noise than realistic images due to the nature of the acquisition devices. This of course poses great challenges to any image segmentation technique. In order to reduce noise, many devises increase the partial voluming, that is, they average acquisition on a thick slice. This leads to blurring the edges between the objects, which make decisions very hard for automatic tools [1]. The different acquisition modalities, the different image manipulations and variability of organs all contribute to a large verity of medical images. It can be safely said that there is no single image segmentation method that suits all possible images. This can pose great problems for any segmentation method. Therefore, several types of image segmentation techni- ques [3-8] were found to achieve accurate segmentations. Among them, the fuzzy clustering methods are of considerable benefits for MRI brain image segmentation $[9,10]$ because the uncertainty of MRI image is widely presented in data. Also the MRIs contain weak boundaries i.e. pixels inside the region and on the boundaries have similar intensity; this causes difficulty working with methods based on edge detection techniques $[7,8]$. The fuzzy c-means clustering algorithms fall into two categories: fuzzy c-means (FCM) [9] and possibilistic c-means (PCM) [10]. Many extensions of the FCM algorithm have been proposed to overcome above fuzzy clustering problem and reduce errors in the segmentation process [9-13]. There are also other methods for enhancing the FCM performance. For example, to improve the segmentation performance, one can combine the pixel-wise classification with pre-processing (noise cleaning in the original image) $[11,13]$ and post-processing (noise cleaning on the classified data). Xue et al. [13] proposed an algorithm where they firstly denoise images and then classify the pixels using the standard FCM method. These methods can reduce the noise to a certain extent, but still have some drawbacks such as increasing com- 
putational time, complexity and introducing unwanted smoothing [14,15]. Liew et al. [16] proposed a spatial FCM clustering algorithm for clustering and segmenting the images by using both the feature space and spatial information. Another variant of FCM algorithm called the robust fuzzy c-means (RFCM) algorithm was proposed in [17].

Pham and Prince [18] modified the FCM objective function by introducing a spatial penalty for enabling the iterative algorithm to estimate spatially smooth membership functions. Ahmed et al. [8] introduced a neighborhood averaging additive term into the objective function of FCM. They named the algorithm bias corrected FCM (BCFCM). Liew and Yan [19] introduced a spatial constraint to a fuzzy cluster method where the inhomogeneity field was modeled by a B-spline surface. The spatial voxel connectivity was implemented by a dissimilarity index, which enforced the connectivity constraint only in the homogeneous areas. This way preserves significantly the tissue boundaries. Zanaty and Aljahdali [11] introduced a new local similarity measure by combining spatial and gray level distances. They used their method as an alternative pre-filtering to an enhanced fuzzy c-means algorithm (EnFCM) [20]. Kang et al. [21] proposed a spatial homogeneity-based FCM (SHFCM). Wang et al. [22] incorporated both the local spatial context and the non-local information into the standard FCM cluster algorithm. They used a novel dissimilarity measure in place of the usual distance metric. In those methods, effect of noise can be overcome by incorporating possibility (typicality) function in addition to membership function. For that, the possibilistic c-means algorithm (PCM) was developed in [23]. This technique combines FCM and logic with some modification in its membership function for removal of noise from the MRI brain images. It has been shown to be more robust to outliers than FCM. However, the robustness of PFCM comes at the expense of the stability of the algorithm [24]. The PCM-based algorithms suffer from the coincident cluster problem that makes them too sensitive to initialization [24]. Many efforts have been presented to improve the stability of possibilistic clustering [25-27].

Although the previous MRIs segmentation algorithms had suppressed the impact of noise and intensity inhomogeneity to some extents, these algorithms still produce misclassified small regions. The problems of over-segmentation and sensitivity to noise are still the challenge. FCM and PCM are also very sensitive to initialization and sometimes coincident clusters will occur. Moreover, coincident clusters may occur during fuzziness processes which can affect the final segmentation.

In this paper, we propose a new method called PFCM which combines the characteristics of both FCM and PCM algorithms by new weights for accurate MRIs segmentation. The proposed method avoids various problems of existing fuzzy clustering methods, solves the noise sensitivity defect of FCM and overcomes the coincident clusters problem of PCM. In order to reduce the noise effect during segmentation, the proposed algorithm combines the objective functions of conventional FCM algorithm and PCM algorithm. To overcome the problem of coincident clusters of PCM and also for combination of the objective FCM and PCM, a new weight function is proposed that is based on Gaussian membership. In this method the effect of noise is overcome by incorporating possibility (typicality) function in addition to membership function. Consideration of these constraints can greatly control the noise in the image as shown in our experiments. The efficiency of the proposed algorithm is demonstrated by extensive segmentation experiments using real MRIs and comprising with other state of the art algorithms.

The rest of this paper is organized as follows: The theoretical foundation of fuzzy c-means and possibilistic c-means is described in Section 2. In Section 3, the proposed PFCM algorithm is presented. Experimental and comparisons results are given in Section 4. Finally, Section 5 gives our conclusions.

\section{FCM and PCM Algorithms}

The FCM algorithm [10] is an iterative clustering method that produces optimal $C$ partitions by minimizing the weighted within the group sum of squared error objective function $J_{F C M}$ :

$$
J_{F C M}(V, U, X)=\sum_{i=1}^{C} \sum_{j=1}^{n} u_{i j}^{m} d^{2}\left(x_{j}, v_{i}\right), 1<m<+\infty
$$

where $X=\left\{x_{1}, x_{2}, \cdots, x_{n}\right\} \subseteq R^{p}$ is the data set in the $p$-dimensional vector space, $n$ is the number of points, $p$ is the number of data items, $C$ is the number of clusters with $2<C<n-1 . V=\left\{v_{1}, v_{2}, \cdots, v_{C}\right\}$ is the $C$ centers or prototypes of the clusters, $v_{i}$ is the $p$-dimension center of the cluster $i$, and $d^{2}\left(x_{j}, v_{i}\right)$ is a square distance measure between object $x_{j}$ and cluster center $v_{i}, U=\left\{u_{i j}\right\}$ represents a fuzzy partition matrix with $u_{i j}=u_{i}\left(x_{j}\right)$ is the degree of membership of $x_{j}$ in the $i^{\text {th }}$ cluster; $x_{j}$ is the $j^{\text {th }}$ of $p$-dimensional measured data. The fuzzy partition matrix satisfies:

$$
\begin{gathered}
0<\sum_{j=1}^{n} u_{i j}<n, \forall i \in\{1, \cdots, C\} \\
\sum_{i=1}^{c} u_{i j}=1, \forall j \in\{1, \cdots, n\}
\end{gathered}
$$

The parameter $m$ is a weighting exponent for each fuzzy membership and determines the amount of fuzzi- 
ness of the resulting classification; it is a fixed number greater than one. The objective function $J_{F C M}$ can be minimized under the constraint of $U$.

The objective function $J_{F C M}$ is minimized with respect to $u_{i j}$ and $v_{i}$, respectively:

$$
\begin{gathered}
u_{i j}=\left[\sum_{k=1}^{c}\left(\frac{d^{2}\left(x_{j}, v_{i}\right)}{d^{2}\left(x_{j}, v_{k}\right)}\right)^{2 l(m-1)}\right]^{-1}, 1 \leq i \leq C, i \leq j \leq n . \\
v_{i}=\frac{\sum_{k=1}^{n} u_{i k}^{m} x_{k}}{\sum_{k=1}^{n} u_{i k}^{m}}, 1 \leq i \leq C .
\end{gathered}
$$

Although FCM and the modified FCM [18,20,22] are useful clustering methods, their memberships do not always correspond well to the degree of belonging of the data, and may be inaccurate in a noisy environment, because the real data unavoidably involves noise. To alleviate weakness of FCM, and to produce memberships that have a good explanation for the degree of belonging of the data. Wang et al. [22] relaxed the constrained condition (3) of the fuzzy $C$-partition to obtain a possibilistic type of membership function and propose PCM for unsupervised clustering. The component generated by the PCM corresponds to a dense region in the data set; each cluster is independent of the other clusters in the PCM strategy. The objective function of the PCM can be formulated as follows:

$$
J_{P C M}(V, U, X)=\sum_{i=1}^{C} \sum_{j=1}^{n} u_{i j}^{m} d^{2}\left(x_{j}, v_{i}\right)+\sum_{i=1}^{C} \eta_{i} \sum_{j=1}^{n}\left(1-u_{i j}\right)^{m}
$$

where

$$
\eta_{i}=\frac{\sum_{j=1}^{n} u_{i j}^{m} d^{2}\left(x_{j}, v_{i}\right)}{\sum_{j=1}^{n} u_{i j}^{m}}
$$

is the scale parameter at the $i^{\text {th }}$ cluster, and

$$
u_{i j}=\frac{1}{1+\left(\frac{d^{2}\left(x_{j}, v_{i}\right)}{\eta_{i}}\right)^{\frac{1}{m-1}}}
$$

is the possibilistic typicality value of training sample $x_{j}$ belonging to the cluster $i, m \in] 1, \infty[$ is a weighting factor called the possibilistic parameter.

Zanaty and Sultan [11] proposed a method for automatic fuzzy algorithms by considering some spatial constraints on the objective function. The algorithm starts of subdivide the data a set of $N$ vector $X=\left\{x_{j}, j=1, \cdots, N\right\}$ into $M$ clusters using well-known fuzzy method [10] (see Equations (1)-(4)). Assume, the data is divided into $M$ cluster, $R_{1}, R_{2}, \cdots, R_{M}$ with centres $v_{1}, v_{2}, \cdots, v_{M}$ respectively. The proposed algorithm processes every two neighbours clusters individually, i.e. if we have three clusters $A, B, C$ with centres $c_{A}, c_{B}$, and $c_{c}$. We start to hold our validity function between clusters $A$ and $B$ if:

$$
\left\|v_{A}-v_{B}\right\|<\left\|v_{A}-v_{c}\right\|
$$

Our validity function is proposed to use the intracluster distance measure, which is simply the distance between a centre of cluster $A$ and cluster centre $B$ multiplied by the objective function of fuzzy. We can define the validity function as:

$$
\begin{aligned}
& V_{1}=\left\|v_{A}-v_{B}\right\|^{2} \frac{1}{n} \sum_{i=1}^{n} u_{i}^{m}\left(d_{i A}+d_{i B}\right) \\
& V_{2}=(\max (A)-\min (B)) \sum_{i=1}^{n} \sum_{k=1}^{2} u_{i k}^{m} d_{i A \cup B}
\end{aligned}
$$

where $\max (B)$ and $\min (B)$ are the maximum and minimum values of clusters $A$ and $B$ respectively. While $d_{A \cup B}$ is the distances of the data $x_{i}$ (of number n) of $A$ union $B$ i.e. $A \cup B$. This algorithm works iteratively the number of clusters increases automatically according to the decision of validity function in Equations (9) and (10), more discussion can be shown in [11].

Typical of other cluster approaches, the PCM also depends on initialization. In PCM technique [25], the clusters do not have a lot of mobility, since each data point is classified as only one cluster at a time rather than all the clusters simultaneously. Therefore, a suitable initialization is required for the algorithms to converge to nearly global minimum.

$$
J_{F P C M}(U, T, V)=\sum_{i=1}^{c} \sum_{j=1}^{n}\left(u_{i j}^{m}+t_{i j}{ }^{\eta}\right) d^{2}\left(x_{j}, v_{i}\right)
$$

with the following constraints:

$$
\begin{aligned}
& \sum_{i=1}^{C} u_{i j}=1, \forall \in\{1, \cdots, n\} \\
& \sum_{j=1}^{n} t_{i j}=1, \forall i \in\{1, \cdots, C\}
\end{aligned}
$$

A solution of the objective function can be obtained via an iterative process where the degrees of membership, typicality and the cluster centers are updated as follows:

$$
\begin{gathered}
u_{i j}=\left[\sum_{k=1}^{C}\left(\frac{d^{2}\left(x_{j}, v_{i}\right)}{d^{2}\left(x_{j}, v_{k}\right)}\right)^{2 /(m-1)}\right]^{-1}, 1 \leq i \leq C, 1 \leq j \leq n \\
t_{i j}=\left[\sum_{k=1}^{n}\left(\frac{d^{2}\left(x_{j}, v_{i}\right)}{d^{2}\left(x_{j}, x_{k}\right)}\right)^{2 /(\eta-1)}\right]^{-1}, 1 \leq i \leq C, 1 \leq j \leq n
\end{gathered}
$$




$$
v_{i}=\frac{\sum_{k=1}^{n}\left(u_{i k}^{m}+t_{i k}^{\eta}\right) x_{k}}{\sum_{k=1}^{n}\left(u_{i k}^{m}+t_{i k}^{\eta}\right)}, 1 \leq i \leq C
$$

Pal et al. [26] improved this method by adding a new penalty to the objective function in order to control the noise affects. The objective unction can be written as:

$$
\begin{gathered}
J_{F P C M}(U, T, V)=\sum_{i=1}^{c} \sum_{j=1}^{n}\left(u_{i j}^{m}+t_{i j}{ }^{\eta}\right) d^{2}\left(x_{j}, v_{i}\right) \\
+\sum_{i=1}^{c} \gamma_{i} \sum_{j=1}^{n}\left(1-t_{i j}\right)^{\eta} \\
\sum_{i=1}^{C} u_{i j}=1, \forall j \in\{1, \cdots, n\} \\
\sum_{j=1}^{n} t_{i j}=1, \forall i \in\{1, \cdots, C\}
\end{gathered}
$$

with the following constraints:

$$
\begin{aligned}
& u_{i j}=\left[\sum_{k=1}^{C}\left(\frac{d^{2}\left(x_{j}, v_{i}\right)}{d^{2}\left(x_{j}, v_{k}\right)}\right)^{2 /(m-1)}\right]^{-1}, 1 \leq i \leq C, 1 \leq j \leq n \\
& t_{i j}=\frac{1}{1+\left(d_{i j}^{2} / \gamma_{i}\right)^{1 /(\eta-1)}} \\
& v_{i}=\frac{\sum_{k=1}^{n}\left(u_{i k}^{m}+t_{i k}^{\eta}\right) x_{k}}{\sum_{k=1}^{n}\left(u_{i k}^{m}+t_{i k}^{\eta}\right)}, 1 \leq i \leq C \\
& \gamma_{i}=\frac{Z \sum_{j=1}^{n} u_{i j}^{m} d_{i j}^{2}}{\sum_{j=1}^{n} u_{i j}^{m}}, Z>1
\end{aligned}
$$

Rajendran and Dhanasekaran [24] define a clustering algorithm that combines the characteristics of both fuzzy and possibilistic c-means. Memberships and typicalities are important for the correct feature of data substructure in clustering problems. Thus, an objective function in this method that depends on both memberships and typicalities can be shown as:

$$
\begin{aligned}
& J_{F P C M}(U, T, V) \\
& =\sum_{i=1}^{c} \sum_{j=1}^{n}\left(a u_{i j}^{m}+b t_{i j}^{\eta}\right) d^{2}\left(x_{j}, v_{i}\right) \\
& \quad+\sum_{i=1}^{c} \gamma_{i} \sum_{j=1}^{n}\left(1-t_{i j}\right)^{\eta}
\end{aligned}
$$

with the following constraints:

$$
\begin{aligned}
& \sum_{i=1}^{C} u_{i j}=1, \forall j \in\{1, \cdots, n\} \text { and } a, b>0 \\
& \sum_{j=1}^{n} t_{i j}=1, \forall i \in\{1, \cdots, C\}
\end{aligned}
$$

A solution of the objective function can be obtained via an iterative process where the degrees of membership, typicality and the cluster centers are updated as follows:

$$
\begin{gathered}
u_{i j}=\left[\sum_{k=1}^{C}\left(\frac{d^{2}\left(x_{j}, v_{i}\right)}{d^{2}\left(x_{j}, v_{k}\right)}\right)^{2 /(m-1)}\right]^{-1}, 1 \leq i \leq C, 1 \leq j \leq n \\
t_{i j}=\frac{1}{1+\left(b d_{i j}^{2} / \gamma_{i}\right)^{1 /(n-1)}} \\
v_{i}=\frac{\sum_{k=1}^{n}\left(a u_{i k}^{m}+b t_{i k}^{\eta}\right) x_{k}}{\sum_{k=1}^{n}\left(a u_{i k}^{m}+b t_{i k}^{\eta}\right)}, 1 \leq i \leq C
\end{gathered}
$$

The above equations indicate that membership $u_{i j}$ is influenced by all $C$ cluster centers, while possibility $t_{i j}$ is influenced just by the $i^{\text {th }}$ cluster center $c_{i}$. The possibilistic term distributes the $t_{i j}$ with respect to every $n$ data points, but not by means of every $C$ clusters. Thus, membership can be described as relative typicality, it determines the degree to which a data fit in to cluster in accordance with other clusters and is helpful in correctly labeling a data point. Possibility can be observed as absolute typicality, it determines the degree to which a data point belongs to a cluster correctly, it can decrease the consequence of noise. Joining both membership and possibility can yield to good clustering result [28].

\section{The Proposed PFCM Algorithm}

The choice of an appropriate objective function is a key to the success of cluster analysis and to obtain better quality clustering results; hence, clustering optimization is based on the objective function [28]. To identify a suitable objective function, one may start from the following set of requissrements: the distance between the data points assigned to a cluster should be minimized and the distance between clusters should to be maximized [26]. To obtain an appropriate objective function, we take into consideration the following:

- The distance between clusters and the data points allocated to them must be reduced.

- Coincident clusters may occur and must to be controlled.

- Selecting the initialization sensitive parameters for decreasing noises affect. 
The desirability between data and clusters is modeled by the objective function. Hung et al. [29] provides a modified PCM technique which considerably improves the function of FCM because of a prototype-driven learning of parameter. The learning procedure of is dependent on an exponential separation strength between clusters and is updated at every iteration.

As for the common value used for this parameter by every data for iterations, we propose a new weight function $w_{i j}$ which is based on Gaussian membership of a point $p$. achieving every point of the data set has a weight in relation to every cluster. The usage of weights produces good classification particularly in the case of noisy data. The weight is calculated as follows:

$$
w_{i j}=\frac{1}{\sqrt{2 \Pi} \sigma_{i}} \mathrm{e}^{\frac{-\left(p_{j}-\mu_{i}\right)^{2}}{2 \sigma_{i}^{2}}}
$$

where $\mu_{i}=\frac{\sum_{j=1}^{n} x_{j}}{n}, \sigma_{i}^{2}=\frac{\sum_{j=1}^{n}\left(p_{j}-\mu_{i}\right)}{n}$ and $w_{i j}$ is the weight of the point $j$ in relation to class $i$. This weight is used to modify the fuzzy and typical partition. To classify a data point, the cluster centroid has to be closest to the data point, it is membership; and for estimating the centroids, the typicality is used for alleviating the undesirable effect of outliers. The objective function is composed of two expressions: the first is the fuzzy function and it uses a fuzziness weighting exponent, the second is possibililstic function and it uses a typical weighting exponent; but the two coefficients in the objective function are only used as exhibitor of membership and typicality. A new relation, lightly different, enabling a more rapid decrease in the function and increase in the membership and the typicality when they tend toward 1 and decrease this degree when they tend toward 0 . This relation is to add weighting Gaussian exponent as exhibitor of distance in the two under objective functions.

To solve the noise sensitivity defect of FCM which has an influence on the estimation of centroids, and to overcome the coincident clusters problem of PCM, the hybridization of possibilistic c-means (PCM) and fuzzy c-means $(\mathrm{FCM})$ is proposed that often avoids various problems. We will use the proposed weight $w_{i j}$ and the term $\left(1-t_{i j}\right)^{\eta}$ in the objective function for alleviating the noise affect and to decrease the distances between clusters and centers and to avoid coincident clusters. Thus the objective function of the proposed method (PFCM) can be formulated as follows:

$$
\sum_{i=1}^{C} u_{i j}=1, \forall j \in\{1, \cdots, n\}, t_{i j} \leq 1,1 \leq j \leq n, 1 \leq i \leq C
$$

$$
\begin{aligned}
L_{m}= & \sum_{i=1}^{C} \sum_{j=1}^{N}\left(u_{i j}^{m}+t_{i j}^{m}\right) w_{i j}^{m} d\left(x_{j}, v_{i}\right)^{2 m} \\
& +\sum_{i=1}^{C} \sum_{j=1}^{n} u_{i j}^{m}\left(1-t_{i j}\right)^{\eta} w_{i j}^{\eta} d\left(x_{j}, v_{i}\right)^{2 m}
\end{aligned}
$$

where $\eta \geq 1$ and $\eta$ is a user defined constants and the parameter $m$ is a weighting exponent for each fuzzy membership. More datasets are tested in [30], they proved that there is a relation between the data shape and $m$. For instance, the triangular shape will fit better if $m=$ 3 is used, more discussion can be found in [10]. Therefore we take into account the data shape in the objective function and to be general for all tested data sets. This penalty term also contains spatial neighborhood information, which acts as a regularizer and biases the solution toward piecewise-homogeneous labeling. Such regularization is helpful in segmenting images corrupted by noise. The objective function $J_{m}$ under the constraint of $u_{i j}$ and $v_{i}$ can be obtained by using the following theorem [25]:

Theorem: Let $X=\left\{x_{i}, i=1,2, \cdots, N \mid x_{i} \in R^{d}\right\}$ denotes an image with $n$ pixels to be partitioned into $C$ classes (clusters), where $x_{i}$ represents feature data. The algorithm is an iterative optimization that minimizes the objective function defined by Equation (23) with the constraints $\sum_{i=1}^{C} u_{i j}=1, \forall j \in\{1, \cdots, n\}$. Then $u_{i j} t_{i j}$ and $v_{i}$ must satisfy the following equalities:

$$
\begin{aligned}
& u_{i j}=\left[\frac{\sum_{i=1}^{c} w_{i j}^{m} d\left(x_{j}, v_{i}\right)^{2 m}+\left(1-t_{i j}\right)^{\eta} w_{i j}^{\eta} d\left(x_{j}, v_{i}\right)^{2 m}}{w_{i j}^{m} d\left(x_{j}, v_{i}\right)^{2 m}+\left(1-t_{i j}\right)^{\eta} w_{i j}^{\eta} d\left(x_{j}, v_{i}\right)^{2 m}}\right]^{\frac{1}{m-1}} \\
& v_{i}=\left(\frac{\left(\sum_{i=1}^{c} \sum_{j=1}^{n}\left(u_{i j}^{m}+t_{i j}^{m}\right) w_{i j}^{m}+\sum_{i=1}^{c} \gamma_{i} \sum_{j=1}^{n}\left(1-t_{i j}\right)^{m}\right) x_{j}}{\sum_{i=1}^{c} \sum_{j=1}^{n}\left(u_{i j}^{m}+t_{i j}^{m}\right) w_{i j}^{m}+\sum_{i=1}^{c} \gamma_{i} \sum_{j=1}^{n}\left(1-t_{i j}\right)^{m}}\right)^{\frac{1}{2 m-1}} \\
& t_{i j}=\left(\frac{\sum_{i=1}^{c} \sum_{j=1}^{n} u_{i j}^{m} w_{i j}^{\eta} d\left(x_{j}, v_{i}\right)^{2 m}}{\sum_{i=1}^{c} \sum_{j=1}^{n} w_{i j}^{m} d\left(x_{j}, v_{i}\right)+\sum_{i=1}^{c} \sum_{j=1}^{n} u_{i j}^{m} w_{i j}^{m} d\left(x_{j}, v_{i}\right)^{2 m}}\right)^{\frac{1}{\eta-1}}
\end{aligned}
$$

Proof: The minimization of constraint problem $J_{m}$ in Equation (23) under the given constraints can be solved of using the Lagrange multiplier method. We define a new objective function with the constraint condition of (Equation (23)) as follows: 


$$
\begin{aligned}
& L_{m}=\sum_{i=1}^{C} \sum_{j=1}^{N}\left(u_{i j}^{m}+t_{i j}^{m}\right) w_{i j}^{m} d\left(x_{j}, v_{i}\right)^{2 m} \\
& +\sum_{i=1}^{c} \sum_{j=1}^{n} u_{i j}^{m}\left(1-t_{i j}\right)^{\eta} w_{i j}^{\eta} d\left(x_{j}, v_{i}\right)^{2 m}+\sum_{i=1}^{n} \lambda_{i}\left(1-\sum_{i=1}^{c} u_{i j}\right)
\end{aligned}
$$

Taking the partial derivative of $L_{m}$ with respect to $u_{k i}$ and $\lambda_{i}$ and then setting them to equal to zero, we have:

$$
\begin{aligned}
& \frac{\partial L_{m}}{\partial U_{m}}=0 \Rightarrow m u_{i j}^{m-1} w_{i j}^{m} d\left(x_{j}, v_{i}\right)^{2 m} \\
& +m u_{i j}^{m-1}\left(1-t_{i j}\right)^{\eta} w_{i j}^{\eta} d\left(x_{j}, v_{i}\right)^{2 m}+\lambda_{i}(-1)=0
\end{aligned}
$$

$$
\frac{\partial L_{m}}{\partial \lambda_{i}}=0 \Rightarrow \sum_{k=1}^{C} u_{k i}-1=0
$$

From Equation (27), we get:

$$
u_{i j}=\left(\frac{\lambda_{i}}{m\left(w_{i j}^{m} d\left(x_{j}, v_{i}\right)^{2 m}+\left(1-t_{i j}\right)^{\eta} w_{i j}^{\eta} d\left(x_{j}, v_{i}\right)^{2 m}\right)}\right)^{\frac{1}{m-1}}
$$

By substitution from Equation (29) into Equation (28), we get

$$
\begin{aligned}
& \left(\frac{\lambda_{i}}{m}\right)^{\left(\frac{1}{m-1}\right)} \sum_{i=1}^{C}\left(\frac{1}{\left(w_{i j}^{m} d\left(x_{j}, v_{i}\right)^{2 m}+\left(1-t_{i j}\right)^{\eta} w_{i j}^{\eta} d\left(x_{j}, v_{i}\right)^{2 m}\right)}\right)^{\left(\frac{1}{m-1}\right)}=1 \\
& \left(\frac{\lambda_{i}}{m}\right)^{\left(\frac{1}{m-1}\right)}=\frac{1}{\sum_{i=1}^{C}\left(\left(w_{i j}^{m} d\left(x_{j}, v_{i}\right)^{2 m}+\left(1-t_{i j}\right)^{\eta} w_{i j}^{\eta} d\left(x_{j}, v_{i}\right)^{2 m}\right)\right)^{\left(\frac{-1}{m-1}\right)}} \\
& u_{i j}=\left[\frac{\sum_{i=1}^{c} w_{i j}^{m} d\left(x_{j}, v_{i}\right)^{2 m}+\left(1-t_{i j}\right)^{\eta} w_{i j}^{\eta} d\left(x_{j}, v_{i}\right)^{2 m}}{w_{i j}^{m} d\left(x_{j}, v_{i}\right)^{2 m}+\left(1-t_{i j}\right)^{\eta} w_{i j}^{\eta} d\left(x_{j}, v_{i}\right)^{2 m}}\right]^{\frac{1}{m-1}} \\
& \frac{\partial L_{m}}{\partial t_{i k}}=0 \Rightarrow \eta \sum_{i=1}^{c} \sum_{j=1}^{n} t_{i j}^{\eta-1} w_{i j}^{m} d\left(x_{j}, v_{i}\right)^{2 m}-\eta \sum_{i=1}^{c} \sum_{j=1}^{n} u_{i j}^{m}\left(1-t_{i j}\right)^{\eta-1} w_{i j}^{\eta} d\left(x_{j}, v_{i}\right)^{2 m}=0 \\
& \frac{\partial L_{m}}{\partial v_{i}}=0 \Rightarrow-2 m \sum_{i=1}^{c} \sum_{j=1}^{n}\left(u_{i j}^{m}+t_{i j}^{m}\right) w_{i j}^{m}\left\|x_{j}-v_{i}\right\|^{2 m-1}-2 m \sum_{i=1}^{c} \sum_{j=1}^{n} u_{i j}^{m}\left(1-t_{i j}\right)^{\eta} w_{i j}^{\eta}\left\|x_{j}-v_{i}\right\|^{2 m-1}=0 \\
& t_{i j}=\left(\frac{\sum_{i=1}^{c} \sum_{j=1}^{n} u_{i j}^{m} w_{i j}^{\eta} d\left(x_{j}, v_{i}\right)^{2 m}}{\sum_{i=1}^{c} \sum_{j=1}^{n} w_{i j}^{m} d\left(x_{j}, v_{i}\right)+\sum_{i=1}^{c} \sum_{j=1}^{n} u_{i j}^{m} w_{i j}^{m} d\left(x_{j}, v_{i}\right)^{2 m}}\right)^{\frac{1}{\eta-1}} \\
& v_{i}=\left(\frac{\left(\sum_{i=1}^{c} \sum_{j=1}^{n}\left(u_{i j}^{m}+t_{i j}^{m}\right) w_{i j}^{m}+\sum_{i=1}^{c} \gamma_{i} \sum_{j=1}^{n}\left(1-t_{i j}\right)^{m}\right) x_{j}}{\sum_{i=1}^{c} \sum_{j=1}^{n}\left(u_{i j}^{m}+t_{i j}^{m}\right) w_{i j}^{m}+\sum_{i=1}^{c} \gamma_{i} \sum_{j=1}^{n}\left(1-t_{i j}\right)^{m}}\right)^{\frac{1}{2 m-1}}
\end{aligned}
$$

The process of finding the best clusters is continued to update the centres $c_{i}$ and the membership $u_{i j}$ using Equations (30) and (31), respectively.

The algorithm for carrying out PFCM for segmentation of MRI brain images can now be stated from the following steps:

1) Select the number of clusters " $C$ " and fuzziness factor " $m$ "
2) Select initial class center prototypes $v=\left\{v_{i}\right\}$; $i=1,2, \cdots, C$, randomly and $\varepsilon$, a very small number

3) Find the value of $w_{i j}$ using Equation (22)

4) Update membershisp function $u_{i j}$ using Equation (30)

5) Update membership function $t_{i j}$ using Equation (31)

6) Update cluster center $v_{i}$ using Equation (32)

7) Repeat steps 3 to 6 until termination $B$, where " $t$ "' 
is the iteration steps, $\|$.$\| is the Euclidean distance norm.$ 8) Stop

\section{Experimental Results}

The experiments were performed on three different sets T1-weigthed and T2-weighted MRIs: in the first test, we used T1-weigthed and T2-weighted at various noise levels $(0 \%, 3 \%, 7 \%$ and $9 \%)$ and radio frequency (RF) levels $(0 \%$ and $20 \%)$ as shown in Figure 1. T1-weigthed corrupted by $6 \%$ salt and pepper noise and the image size is $129 \times 129$ pixels [30] (see Figure 2) is used in the next. The third set includes simulated volumetric MRI consisting of ten classes as shown in Figure 3. The advantages of using digital phantoms rather than real image data for soft segmentation methods include prior knowledge of the true tissue types and control over image parameters such as modality, slice thickness, noise, and intensity in homogeneities. Through our implementation, we set the following parameters: $m=2, \varepsilon=0.0001$ and $\eta=2$. The quality of the segmentation algorithm is of vital importance to the segmentation process. The comparison score $S$ for each algorithm as proposed in [31] is defined as follows:

$$
S=\left|\frac{A \cap A_{\text {ref }}}{A \bigcup A_{\text {ref }}}\right|
$$

where $A$ represents the set of pixels belonging to a class as found by a particular method and $A_{\text {ref }}$ represents the

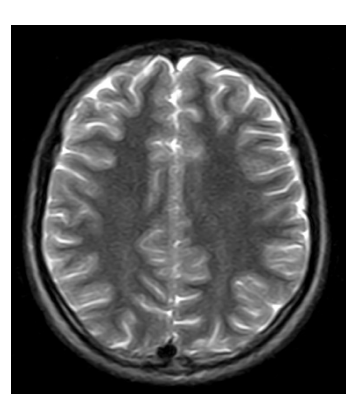

(a)

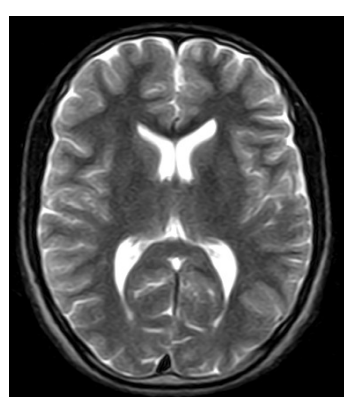

(b)
Figure 1. MRI image: (a) Original T1-weigthed, (b) Original T2-weigthed.

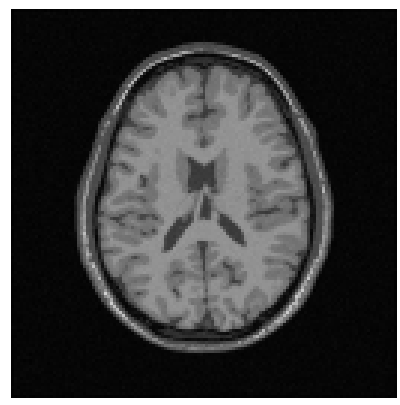

Figure 2. Original T1-weigthed (slice 91). reference cluster pixels.

\subsection{Noisy T1-Weigthed and T2-Weighted MRI}

The proposed technique is applied to T1-weigthed and T2-weighted MRI [32] (as shown in Figures 1(a) and (b)) at various noise levels $(0 \%, 3 \%, 5 \%$ and $9 \%)$ and $\mathrm{RF}$ levels $(0 \%$ and $20 \%)$. To prove the efficiency of proposed algorithm, several noise levels are added to these data sets, while $S$ (Equation (33)) is evaluated for each segment in T1-weigthed and T2-weighted. Figure 4 shows the segmentation output of T1-weigthed and T2-weighted MRI at noise levels $(0 \%, 3 \%, 5 \%$ and $9 \%)$ and RF levels $(0 \%$ and $20 \%$ ). The average of segmentation accuracy scores (average) for each image is indicated in Table 1. Suppose we have an image containing $v$ segments, the accuracy scores can be computed from: average $=\frac{\sum_{i=1}^{v} S_{i}}{v}$.

Table 1 describes the average of the proposed method when applied to the test images. For example in Figure 4 when noise $=0 \%$ and $\mathrm{RF}=0 \%$, average is equal to $0.98 \%$ and $0.95 \%$ for T1-weigthed and T2-weighted MRI respectively. The obtained results show that the proposed algorithms are very robust to noise and intensity homogeneities and inhomogeneities. According to Zijdenbos [32] statement that average $>0.7$ indicates excellent agreement; the proposed algorithm has desired performance in cortical segmentation.

The best average is achieved for low noise and RF levels, for which values of average are higher than 0.94. According to Table 1, the proposed technique is stable at $88 \%$ at noise level $9 \%$ and RF $20 \%$, this result is satisfactory for segmenting the weak boundary tissues.

\subsection{T1-Weighted MRI Phantom}

We used a high-resolution T1-weighted MRI (with slice thickness of $1 \mathrm{~mm}, 6 \%$ noise and RF $20 \%$ ) obtained from the simulated brain database of McGill University [32] (see Figure 2). In this test, beside evaluating the proposed method and the most recent fuzzy c-means such as: Pal et al. [26], Wang et al. [22], Rajendran and Dhana-

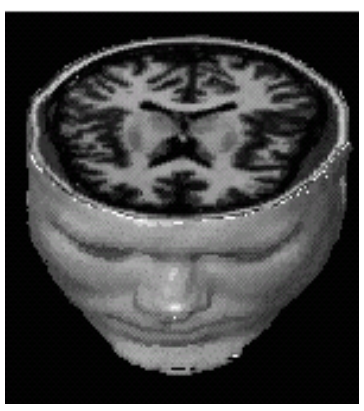

Figure 3. 3D simulated data. 


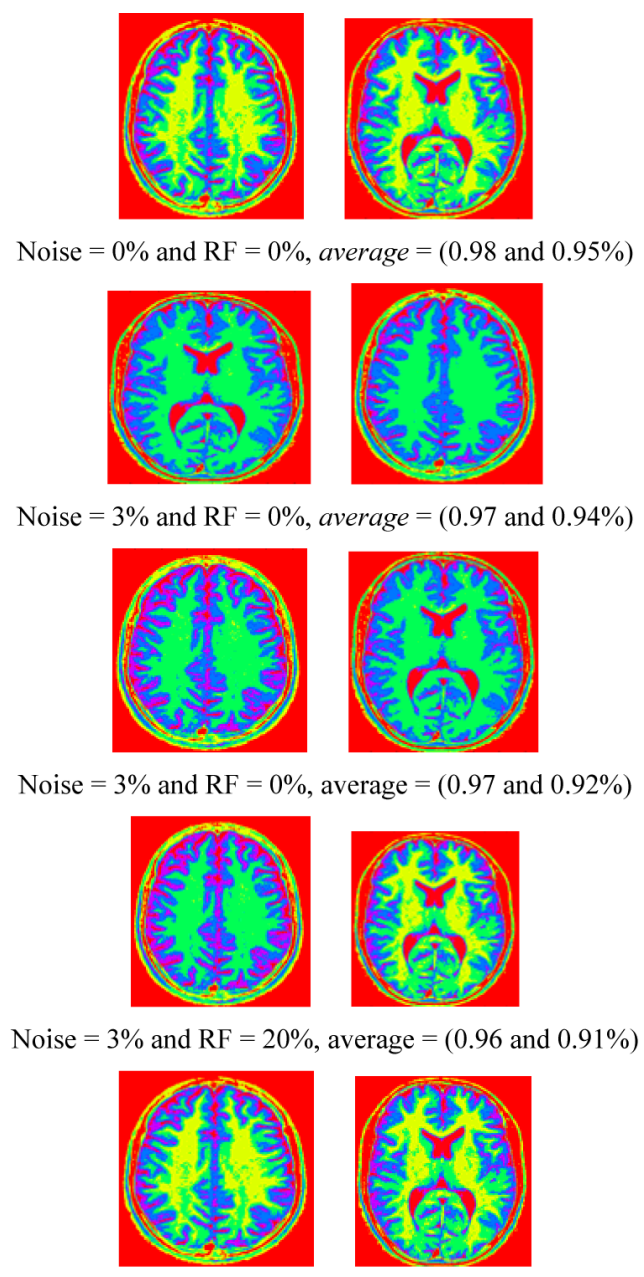

Noise $=5 \%$ and $\mathrm{RF}=0 \%$, average $=(0.95$ and $0.90 \%)$

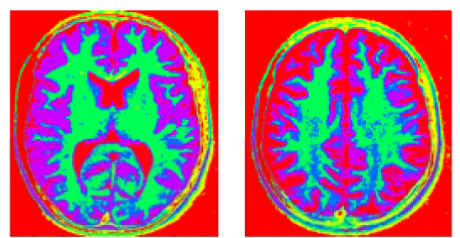

Noise $=5 \%$ and $\mathrm{RF}=20 \%$, average $=(0.93$ and $0.89 \%)$

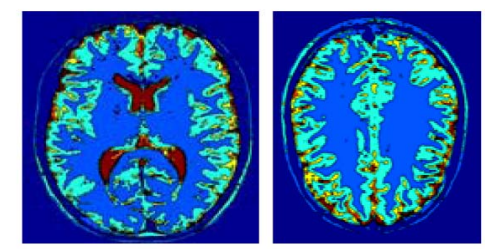

Noise $=9 \%$ and $\mathrm{RF}=0 \%$, average $=(0.92$ and $0.90 \%)$
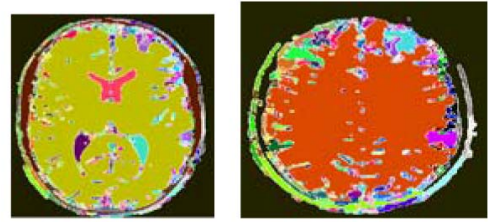

Noise $=9 \%$ and $\mathrm{RF}=20 \%$, average $=(0.90$ and $0.88 \%)$

Figure 4. The segmentation results of T1-weigthed and T2-weighted MRI.
Table 1. The segmentation average of slices (T1-weigthed and T2-weighted MRI) with different situations of noise level and intensity non-uniformity (RF).

\begin{tabular}{ccccc}
\hline$R F$ & \multicolumn{2}{c}{0} & \multicolumn{2}{c}{ 20\% } \\
\cline { 2 - 5 } Noise & \multicolumn{2}{c}{ Slices } & \multicolumn{2}{c}{ Slices } \\
\cline { 2 - 5 } & $\# 51$ & $\# 10$ & $\# 51$ & $\# 10$ \\
\hline $0 \%$ & 0.98 & 0.95 & 0.97 & 0.94 \\
$3 \%$ & 0.97 & 0.92 & 0.96 & 0.91 \\
$5 \%$ & 0.95 & 0.90 & 0.93 & 0.89 \\
$\mathbf{9 \%}$ & 0.92 & 0.90 & 0.90 & 0.88 \\
\hline
\end{tabular}

sekaran [24] and Zanaty and Aljahdali [11] are implemented and applied on the test image to prove the efficiency of the proposed method. Five segments as shown in Figures 5-9 are obtained after applying these methods to this image. Evaluating the accuracy of the existing methods and the proposed method is shown in Table 2. Obviously, the proposed method acquires the best segmentation performance. The proposed method appears to be stable and achieve better performance than Pal et al. [26], Wang et al. [22], Rajendran and Dhanasekaran [24] and Zanaty and Aljahdali [11] by factor $12 \%, 8 \%, 7 \%$, and $3 \%$ respectively.

\subsection{Simulated MR Data}

In this section, we experiment the proposed method and some existing methods such as: Pal et al. [26], Wang et al. [22], Rajendran and Dhanasekaran [24] and Zanaty and Aljahdali [11] when applied to the nine classes (slices\# 51-59) from 3D simulated data (see Figure 3). Table 3 shows the corresponding accuracy scores (\%) of the proposed and the existing methods for the nine classes. Obviously, the FCM gives the worst segmentation accuracy for all classes, while other methods give satisfactory results. On the other hand, the method of Pal et al. [26], Wang et al. [22], Rajendran and Dhanasekaran [24] and Zanaty and Aljahdali [11] acquire the good segmentation performance in case of classes $9,3,4$, and 8 respectively. Overall, the proposed method is more stable and achieves much better performance than the others in all different classes even with misleading of true tissue of validity indexes.

\section{Conclusion}

This paper has presented a new approach called possiblsistic fuzzy c-means (PFCM) which combines FCM and PCM to overcome the weakness of both methods. The proposed algorithm is formulated by modifying the objective function of PCM algorithm to allow the labeling of a pixel to be influenced by other pixels and to suppress the 


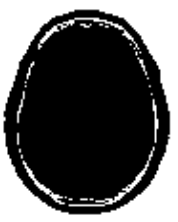

(a)

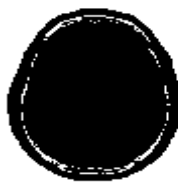

(a)

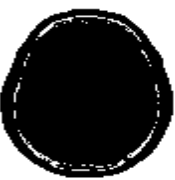

(a)

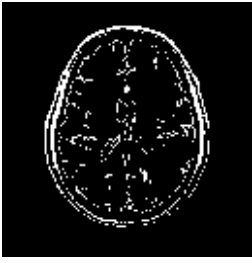

(b)

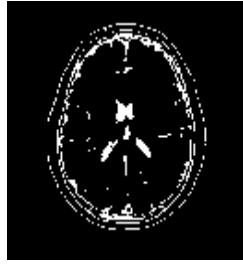

(c)

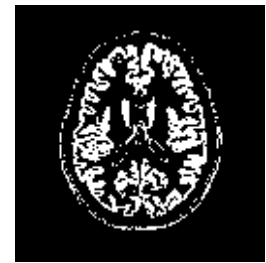

(d)

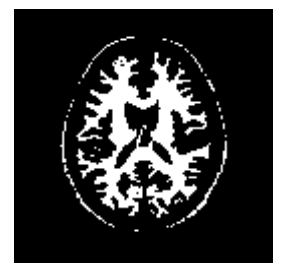

(e)

Figure 5. Results of segmentation using Pal et al. [26].

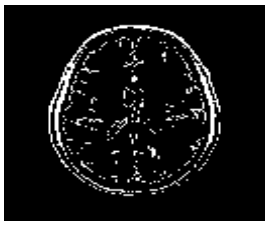

(b)

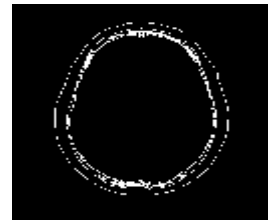

(c)

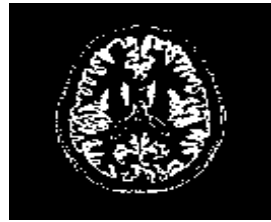

(d)

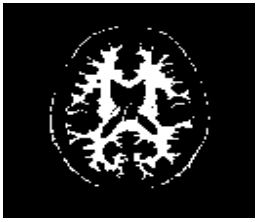

(e)

Figure 6. Results of segmentation using Wang et al. [22].

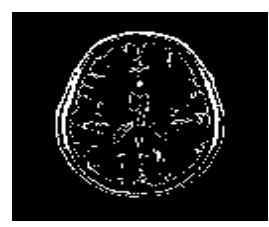

(b)

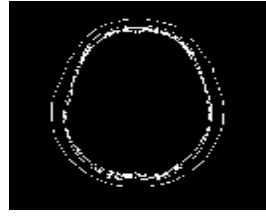

(c)

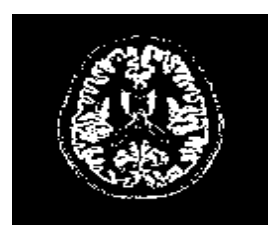

(d)

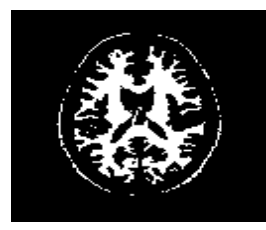

(e)

Figure 7. Results of segmentation using Rajendran and Dhanasskaran method [24].

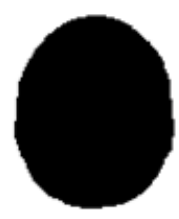

(a)

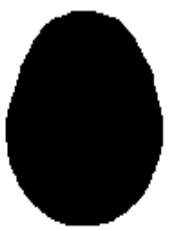

(a)

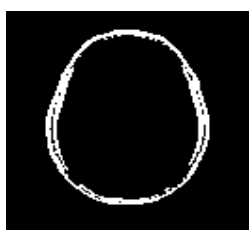

(b)

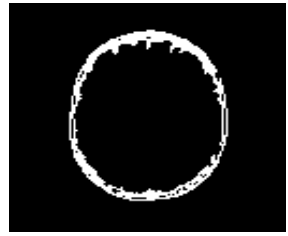

(c)

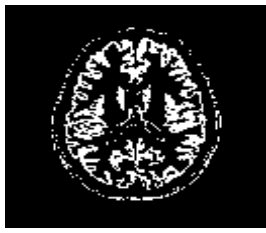

(d)

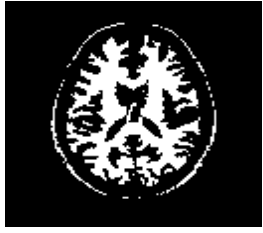

(e)

Figure 8. Results of segmentation Zanaty and Ajahdali [11].

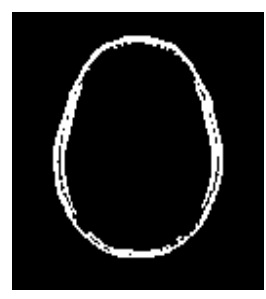

(b)

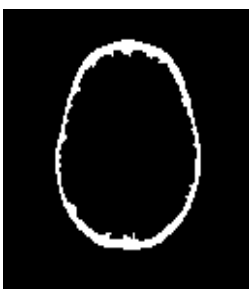

(c)

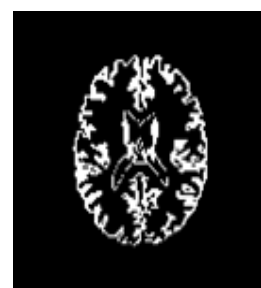

(d)

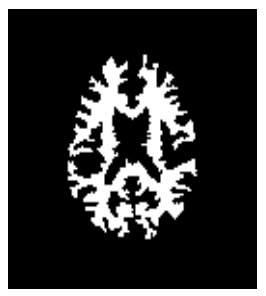

(e)

Figure 9. Results of segmentation using the proposed method.

noise effect during segmentation. To prove the efficiency of the proposed algorithm in segmenting the MRI images, the proposed algorithm has been applied to different data sets. The first set includes T1-weigthed and T2-weighted MRI at noise levels $(0 \%, 3 \%, 5 \%, 7 \%$, and $9 \%)$ and RF levels $(0 \%$ and $20 \%)$. We have noted that the proposed algorithm succeeded to segment noisy MRI images (at noise levels from $0 \%$ to $9 \%$ and RF levels from $0 \%$ to $20 \%$ ). The accuracy average of output segmentation of T1-weigthed and T2-weighted MRI shows excellent performances exceeding $88 \%$ for complex image structures. Next test, we have experimented the proposed algorithm using T1-weigthed MRI with 6\% noise while the score accuracy of each segment is evaluated. The superiority of 
Table 2. Accuracy of the segmentation results.

\begin{tabular}{|c|c|c|c|c|c|c|}
\hline \multirow{2}{*}{ Methods } & \multicolumn{5}{|c|}{ Segments } & \multirow{2}{*}{ Averag } \\
\hline & $\mathrm{a}$ & $\mathrm{b}$ & $\mathrm{c}$ & $\mathrm{d}$ & $\mathrm{e}$ & \\
\hline Pal et al. [26] & $99.2 \%$ & $50.12 \%$ & $73.87 \%$ & $71.73 \%$ & $80.39 \%$ & $75.08 \%$ \\
\hline Wang et al. [22] & $99.0 \%$ & $40.55 \%$ & $87.23 \%$ & $85.0 \%$ & $79.65 \%$ & $80 \%$ \\
\hline Rajendran and Dhanasekaran [24] & $99.1 \%$ & $73.98 \%$ & $79.54 \%$ & $66.54 \%$ & $77.98 \%$ & $79.43 \%$ \\
\hline Zanaty Aljahdali [11] & $99 \%$ & $80.07 \%$ & $83.89 \%$ & $74 \%$ & $81.23 \%$ & $84 \%$ \\
\hline The proposed method & $99.88 \%$ & $77.47 \%$ & $84.86 \%$ & $88.43 \%$ & $87.64 \%$ & $87.66 \%$ \\
\hline
\end{tabular}

Table 3. Segmentation accuracy (\%) of the proposed and the existing methods on brain classes.

\begin{tabular}{|c|c|c|c|c|c|c|c|c|c|c|}
\hline Method & Class 1 & Class 2 & Class 3 & Class 4 & Class 5 & Class 6 & Class 7 & Class 8 & Class 9 & Overall \\
\hline Pal et al. [26] & 68.55 & 63.14 & 82.83 & 78.88 & 73.96 & 67.87 & 96.21 & 23.27 & 97.97 & 72.52 \\
\hline Wang et al. [22] & 80.54 & 70.55 & 83.34 & $86 . .01$ & 83.65 & 87.98 & 88.70 & 27.54 & 93.54 & 76.98 \\
\hline $\begin{array}{c}\text { Rajendran and } \\
\text { Dhanasekaran [24] }\end{array}$ & 78.65 & 68.87 & 69.54 & 95.99 & 85.87 & 71.98 & 73.87 & 66.87 & 94.11 & 78.42 \\
\hline The proposed method & 84.76 & 80.45 & 83.09 & 94.34 & 88.56 & 70.12 & 96.64 & 67.34 & 97.98 & 84.81 \\
\hline
\end{tabular}

the proposed algorithm is demonstrated by comparing its performance against the existing Pal et al. [26], Wang et al. [22], Rajendran and Dhanasekaran [24] and Zanaty and Aljahdali [11]. The proposed method achieves better performance than Pal et al. [26] by factor 12\%, Wang et al. [22] by factor 7\%, Rajendran and Dhanasekaran [24] by factor $8 \%$ and Zanaty and Aljahdali [11] by factor 3\%. In addition, for the simulated 3D data (brain volume consists of ten slices), the average accuracy of the proposed algorithm have been evaluated and compared to Pal et al. [26], Wang et al. [22], Rajendran and Dhanasekaran [24] and Zanaty and Aljahdali [11]. We have noted that the average accuracy of the proposed method gave an improvement about $12 \%, 8 \%, 6 \%$, and $7 \%$ over Pal et al. [26], Wang et al. [22], Rajendran and Dhanasekaran [24] and Zanaty and Aljahdali [11] respectively.

Future research in MRI segmentation should strive toward improving the computation speed of the segmentation algorithms. This is particularly important as MRI imaging is becoming a routine diagnostic procedure in clinical practice. It is also important that any practical segmentation algorithm should deal with 3D volume segmentation instead of 2D slice by slice segmentation, since MRI data is $3 \mathrm{D}$ in nature.

\section{REFERENCES}

[1] R. Bakshi, S. Ariyaratana, R. H. B. Benedict and L. Jacobs, "Fluid-Attenuated Inversion Recovery Magnetic Resonance Imaging Detects Cortical and Juxtacortical Multiple Sclerosis Lesions," Archives of Neurology, Vol.
58, No. 5, 2001, pp. 742-748. http://dx.doi.org/10.1001/archneur.58.5.742

[2] A. Ayman, T. Funatomi, M. Minoh, E. A. Zanaty, T. Okada, K. Togashi, T. Sakai and S. Yamada, "New Region Growing Segmentation Technique for MR Images with Weak Boundaries," IEICE Conference MI2010-79, Japan, 2010, pp. 71-76.

[3] H.-R. Wang, J.-L. Yang, H.-J. Sun, D. Chen and X.-L. Liu, "An Improved Region Growing Method for Medical Image Selection and Evaluation Based on Canny Edge Detection," Management and Service Science (MASS), Wuhan, 12-14 August 2011, pp. 1-4.

[4] M. N. Mubarak, M. M. Sathik, S. Z. Beevi and K. Revathy, "A Hybrid Region Growing Algorithm for Medical Image Segmentation," International Journal of Computer Science \& Information Technology (IJCSIT), Vol. 4, No. 3, 2012.

[5] K. K. L. Wong, J. Y. Tu, R. M. Kelso, S. G. Worthley, P. Sanders, J. Mazumdar and D. Abbott, "Cardiac Flow Component Analysis," Medical Engineering \& Physics, Vol. 32, No. 2, 2010, pp. 174-188. http://dx.doi.org/10.1016/j.medengphy.2009.11.007

[6] E. A. Zanaty, "An Approach Based on Fusion Concepts for Improving Brain Magnetic Resonance Images (MRIs) Segmentation," Journal of Medical Imagining and Health Informatics, Vol. 3, No. 1, 2013, pp. 30-37. http://dx.doi.org/10.1166/jmihi.2013.1122

[7] E. A. Zanaty and A. S. Ghiduk, "A Novel Approach for Medical Image Segmentation Based on Genetic and Seed Region Growing Algorithms," Journal of Computer Science and Information Systems, Vol. 10, No. 3, 2013.

[8] E. A. Zanaty and A. Afifi, "A Watershed Approach for Improving Medical Image Segmentation," Computer Me- 
thods in Biomechanics and Biomedical Engineering, Vol. 16, No. 12, 2012, pp. 1262-1272.

[9] M. N. Ahmed, S. M. Yamany, N. Mohamed, A. A. Farag and T. Moriarty, "A Modified Fuzzy c-Means Algorithm for Bias Field Estimation and Segmentation of MRI Data," IEEE Transactions on Medical Imaging, Vol. 21, No. 3, 2002, pp. 193-199. http://dx.doi.org/10.1109/42.996338

[10] T. N. Pappas, "An Adaptive Clustering Algorithm for Image Segmentation," IEEE Transactions on Signal Processing, Vol. 40, No. 4, 1992, pp. 901-914. http://dx.doi.org/10.1109/78.127962

[11] E. A. Zanaty and S. Aljahdali, "Automatic Fuzzy Algorithms for Reliable Image Segmentation," International Society for Computers and Their Applications, Vol. 19, No. 3, 2012, pp. 166-175.

[12] Z. M. Wang, Y. C. Soh, Q. Song and K. Sim, "Adaptive Spatial Information-Theoretic Clustering for Image Segmentation," Pattern Recognition Letters, Vol. 42, No. 9, 2009, pp. 2029-2044. http://dx.doi.org/10.1016/j.patcog.2009.01.023

[13] J. H. Xue, A. Pizurica, W. Philips, E. Kerre, R. Van de Walle and I. Lemahieu, "An Integrated Method of Adaptive Enhancement for Unsupervised Segmentation of MRI Brain Images," Pattern Recognition Letters, Vol. 24, No. 15, 2003, pp. 2549-2560. http://dx.doi.org/10.1016/S0167-8655(03)00100-4

[14] K. S. Chuang, H. L. Tzeng, S. Chen, J. Wu and T. J. Chen, "Fuzzy c-Means Clustering with Spatial Information for Image Segmentation," Computerized Medical Imaging and Graphics, Vol. 30, No. 1, 2006, pp. 9-15. http://dx.doi.org/10.1016/j.compmedimag.2005.10.001

[15] S. H. Lee and M. M. Crawford, "Unsupervised Multistage Image Classification Using Hierarchical Clustering with a Bayesian Similarity Measure," IEEE Transactions on Image Processing, Vol.14, No. 3, 2005, pp. 312-320.

[16] A. W. C. Liew, S. H. Leung and W. H. Lau, "Fuzzy Image Clustering Incorporating Spatial Continuity," IEE Proceedings of Vision, Image and Signal Processing, Vol. 147, No. 2, 2000, pp. 185-192.

http://dx.doi.org/10.1049/ip-vis:20000218

[17] S. Roy, H. K. Agarwal, A. Carass, Y. Bai, D. L. Pham and J. L. Prince, "Fuzzy c-Means with Variable Compactness," IEEE International Symposium on Biomedical Imaging, 2008, pp. 452-455.

[18] D. Pham and J. Prince, "Adaptive Fuzzy Segmentation of Magnetic Resonance Images," IEEE Transactions on Medical Imaging, Vol. 18, No. 9, 1999, pp. 737-752. http://dx.doi.org/10.1109/42.802752

[19] A. W. C. Liew and H. Yan, "An Adaptive Spatial Fuzzy Clustering Algorithm for 3-D MR Image Segmentation," IEEE Transactions on Medical Imaging, Vol. 22, No. 9, 2003, pp. 1063-1075.

http://dx.doi.org/10.1109/TMI.2003.816956
[20] L. Szilágyi, S. M. Szilágyi and Z. Benyó, “A Modified FCM Algorithm for Fast Segmentation of Brain MR Images," ICIARLNCS, Vol. 4633, 2007, pp. 866-877.

[21] B. Y. Kang, D. W. Kim and Q. Li, "Spatial HomogeneityBased Fuzzy c-Means Algorithm for Image Segmentation," FSKDLNAI, Vol. 3613, 2005, pp. 462-469.

[22] J. Z. Wang, J. Kong, Y. H. Lu, M. Qi and B. X. Zhang, "A Modified FCM Algorithm for MRI Brain Image Segmentation Using Both Local and Non-Local Spatial Constrains," Computerized Medical Imaging and Graphics, Vol. 31, No. 8, 2008, pp. 685-698. http://dx.doi.org/10.1016/j.compmedimag.2008.08.004

[23] Z. Ji, Q. Sun and D. Xia, “A Modified Possibilistic Fuzzy c-Means Clustering Algorithm for Bias Field Estimation and Segmentation of Brain MR Image," Computerized Medical Imaging and Graphics, Vol. 35, No. 5, 2011, pp. 383-397. http://dx.doi.org/10.1016/j.compmedimag.2010.12.001

[24] A. Rajendran and R. Dhanasekaran, "MRI Brain Image Tissue Segmentation Analysis Using Possibilistic Fuzzy c-Means Method," International Journal on Computer Science and Engineering, Vol. 3, No. 12, 2011, pp. $3832-$ 3836.

[25] N. R. Pal, K. Pal and J. C. Bezdek, "A Mixed c-Means Clustering Model," IEEE International Conference on Fuzzy Systems, Vol. 1, 1997, pp. 11-21.

[26] N. R. Pal, K. Pal, J. M. Keller and J. C. Bezdek, "A Possibilistic Fuzzy c-Means Clustering Algorithm," IEEE Transactions on Fuzzy Systems, Vol. 13, No. 4, 2005, pp. 517-530. http://dx.doi.org/10.1109/TFUZZ.2004.840099

[27] H. Timm, C. Borgelt, C. Doring and R. Kruse, "An Extension to Possibilistic Fuzzy Cluster Analysis," Fuzzy Sets and Systems, Vol. 147, No. 1, 2004, pp. 3-16. http://dx.doi.org/10.1016/j.fss.2003.11.009

[28] J. S. Zhang and Y. W. Leung, "Improved Possibilistic cMeans Clustering Algorithms," IEEE Transactions on Fuzzy Systems, Vol. 12, No. 2, 2004, pp. 209-217. http://dx.doi.org/10.1109/TFUZZ.2004.825079

[29] W. L. Hung, M. Yang and D. Chen, "Parameter Selection for Suppressed Fuzzy c-Means with an Application to MRI Segmentation," Pattern Recognition Letters, Vol. 27, No. 5, 2006, pp. 424-438.

[30] Brain Web, "Simulated Brain Database," Mcconnell Brain Imaging Centre, Montreal Neurological Institute, McGill University. http://brainweb.bic.mni.mcgill.ca/brainweb/

[31] Z. H. Yin, Y. G. Tang, F. C. Sun and Z. Q. Sun, "Fuzzy Clustering with Novel Serable Criterion," Tsinghua Science and Technology, Vol. 11, No. 1, 2006, pp. 50-53. http://dx.doi.org/10.1016/S1007-0214(06)70154-7

[32] A. P. Zijdenbos, "MRI Segmentation and the Quantification of White Matter Lesions," PhD Thesis, Vanderbilt University, Nashville, 1994. 